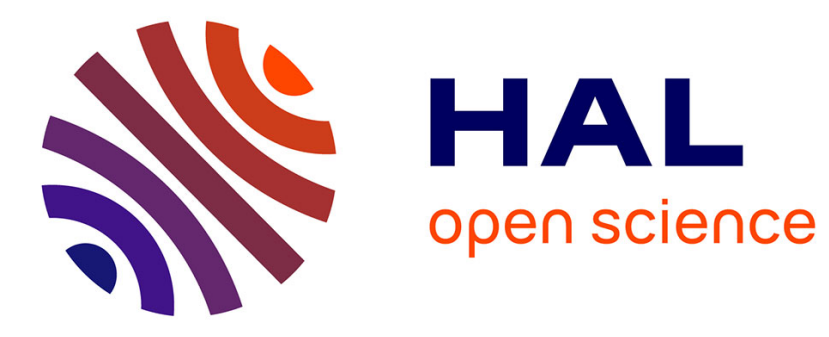

\title{
La dédicace du Trophée des Alpes (La Turbie)
}

Jules Formigé

\section{To cite this version:}

Jules Formigé. La dédicace du Trophée des Alpes (La Turbie). Gallia - Fouilles et monuments archéologiques en France métropolitaine, 1955, 13 (1), pp.101-102. 10.3406/galia.1955.1432 . hal01922590

\section{HAL Id: hal-01922590 \\ https://hal.science/hal-01922590}

Submitted on 6 Feb 2020

HAL is a multi-disciplinary open access archive for the deposit and dissemination of scientific research documents, whether they are published or not. The documents may come from teaching and research institutions in France or abroad, or from public or private research centers.
L'archive ouverte pluridisciplinaire HAL, est destinée au dépôt et à la diffusion de documents scientifiques de niveau recherche, publiés ou non, émanant des établissements d'enseignement et de recherche français ou étrangers, des laboratoires publics ou privés.

\section{(ㅇ)(1) $\$$}

Distributed under a Creative Commons Attribution - NonCommercial - NoDerivatives 44.0 


\section{DOCUMENTS COMMENTÉS}

LA DForcace de Trophéle des ALPES (LA TuRBIE)

La rédaction de Gallia m’a demandé de publier à nouveau ma reconstitution de la dédieace du Trophée des Alpes. Fn eflet, dans les publications oì je m'en suis expliqué 1 , la réduction des reproductions ne permettait pas de juger avec toute la précision désirable du travail accompli. D'autre part, plusieurs fragments ayant été retrouvés et mis en place depuis 1948, il est intéressant de publier l'état actuel (1954) de l'épure originale qui, constamment tenue à jour, est et restera la base de cette reconstitution (fig. 1).

On sait que la dédicace du Trophée est un document privilégić entre tous, puisque son texte nous a été transmis intégralement par Pline l'Ancien dans son Hisloire Nalurelle (III, 136-137). La mise en place sur le monument occupe $17 \mathrm{~m}$. de long sur $3^{\mathrm{m}}, 66$ de haut. Ie découpage en neuf lignes a été dicté par les dimensions des deux séries de caractères et par les fragments de mots qui, précédés ou suivis d'un blanc, représentaient des débuts ou des fins de lignes. La disposition des noms de peuples en suites horizontales (et non en colonnes verticales

(1) Bulletin de la Soc. Nat. des Antiquaires de France, 1943-1944, p. 360-367, pl. V. - Le Trophée des Alpes (La Turbie), supplément II d̀ Gallia, 1949, p. 54-61, flg. 47 (photographie de l'inscription en place). comme l'avait proposé Mommsen au C.I.L., V, 7817) est imposée par certains fragments portant à la fois sur deux lignes. La hauteur respective des assises et la longueur des blocs est donnée par plusieurs fragments et par comparaison avec les autres parties du socle de l'édifice. La disposition régulière de l'appareil ayant été soigneusement établie, le nombre des lettres par blocs a été calculé en fonction d'un écartement moyen des caractères et précisé à l'aide des fragments comportant un joint de bloc. Ciertains fragments donnent les interlignes des petites letlres, égaux à la demilauteur de ces lettres.

Les parties foncées sur le dessin représentent les quelque 140 fragments originaux retrouvés et incrustés dans le panneau moderne, compose de bloes de marbre sur lesquels l'inscription a été regravéc de telle façon que par leur teinte et leur contour, les morceaux antiques se reconnaissent à l'œil nu. La mise en place du texte était assez sûre pour qu'on pût se permettre d'utiliser tous les fragments retrouvés, même ceux (d'ailleurs fort rares) qui, ne portant que sur une partie d'une seule lettre, pouvaient se placer dans l'un ou l'autre exemplaire de cette lettre figurant dans l'inscription. Chaque fois qu'un fragment nouveau est retrouvé, il est donc incrusté à sa place dans le panneau. Et la découverte de 1948 a confirmé l'une des rares divergences de rédaction avec le texte de 
Pline adoptées dans notre reconstitution : la mise en place des lettres sur les blocs nous avait imposé l'abréviation TRIB. (au lieu de TR. donnée par Pline); la découverte du fragment comportant le $B$. nous a donné raison.

Rappelons que les autres divergences, certaines, celles-là, avec la transcription de Pline sont les suivantes:

$1^{\circ}$ le développement en toutes lettres des mots imperatori, Augusto, et Senatus Populusque Romanus, abrígés chez Pline;

$2^{\circ}$ l'orthographe Trumpilini, au lieu de Triumpilini des manuscrits;

$3^{\circ}$ la place des Venostes après les Vennonetes, et non avant.

Jules Formige, Membre de l'Institut.

Liste et localisation des 任 peuples alpestres, de l'Adriatique vers la Méditerranéc lla carte ci-jointe, fig. 2, ne comporte que les localisations les mieux (tssurées) :

Trumpilini, Val 'Trompia, haute vallée de la Mella (affluent de l'Oglio).

Camunsi, Val Camonica, haute vallée de l'Oglio.

Venonetes, Valteline, haute vallée de l'Adda.

Venostes, Val Venosta, haute vallée de l'Adige.

IsARCi, vallée de l'Isarco, aff'luent du haut Adige.

Breuni, région du col du Brenner.

Genaunes, Val Genaun, dans la basse Engadine (vallée de l'Inn).

Focinates : ?

Vindelicorum gentes quattuor : Coschnetes, Rucinates, Licates, Catenates. en Vindélicie, partie occidentale de la province de Rétie. Ies Licates devaient occuper la vallée du Lech (Licus).

Amisontes, haute vallée de la Salzach (Isonta).
Rligusci : ?

Stanetes, entre les hautes vallées du Rhin et de l'Inn (haute Engadinc).

Calucones : ?

Brixentes, sur le Rhin en amont du lac de Constance, région de Bregenz (Brigantium).

Lepont, Val Leventina (hatule vallée du 'Tessin) et $\Lambda$ pes Iépontiennes.

VibER, sources et haute vallée du Rhòne.

Nantuates, sur le Rhòne en amont du Léman, région de Saint-Maurice-enValais (Tarnaiae).

Selptini, sur le Rhône, région de Sion.

Veragri, sur le Rhône, région de Martigny-en-Valais (Octodurus).

Salassi, Val d'Aoste, haute vallée de la Doire Baltée.

Acitavones : ?

Mentels, Maurienne, vallée de l'Arc (affluent de l'Isère).

Jcens, peut-être l'Oisans, vallée de la Romanche.

Catcriges, haute vallée de la Durance. région de Chorges (C'alurigomayus) et d'Embrun.

Brigrani, peut-être la région de Briançon (Brigantio), sources de la Durance.

Sogiontil : ?

Brodioxti = Bodiontici de Digne ?

Nemaloni, Edenates, Estmani (l'Ubaye? ou la Vésubie?), Veamint, Gasdatak, Tridilati, Ectini : ?

Vergicni, peul-être la région de Vergons, dans la vallée du Verdon.

EgVituri, Nenetcri, Oratelli : ?

Nertsi, rapitale Vintium (Vence).

Velalini : ?

Suetri, capitale Salinae (Castellane).

Note de la Rédaction. - La présente note, devant être considérée comme un complément au volume de M. Formigé, Le Trophée des Alpes (Supplément II d̀ Gallia, C.N.R.S., 1949), peut s'acquérir sous couverture au Comité archéologique du C.N.R.S., 155, rue de Sèvres, Paris-XVe. 


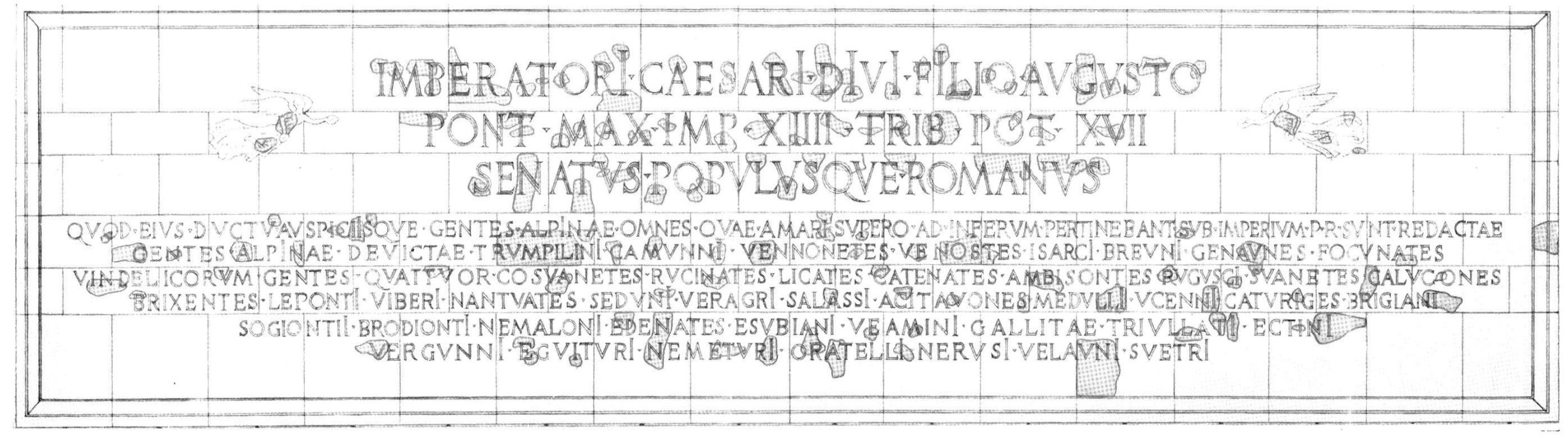

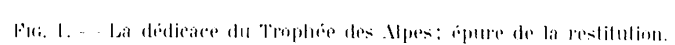

Xon alienum villatur how loco subiecre

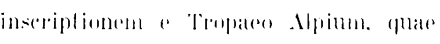
falis oxi:


imp. XIIIJ, tr. port. XVII, S.P.Q.R. queol

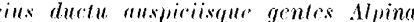

"A l'mpcreur Císar Auguste. vil: du Divin Julrs), grand pontife, imperator pour la XIV frois at revitu de sa XTI


lon $z$ a

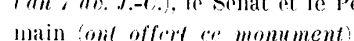

ommess funl, "a mari supero all inforum pertimertam sub imperium P.R. sun



parce quie sous son conmmandement et sers allspires tons les peuples alpins qui se trouraient pntre la Her supérieur

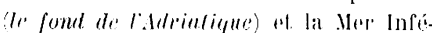

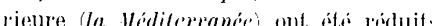
au pouvoir du Peuple Romain.
Triumpilini. Cammuni. Venostes, Vennometes. Isarci, rete....] Suetri.

Xom sunt atdiectae Cottianae rivitate

Pouples alpins: vaintus: les triumpilimi, ate...
Xr. quie non fureant lintiles. item atributie municipiis loge Pompriat

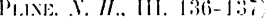




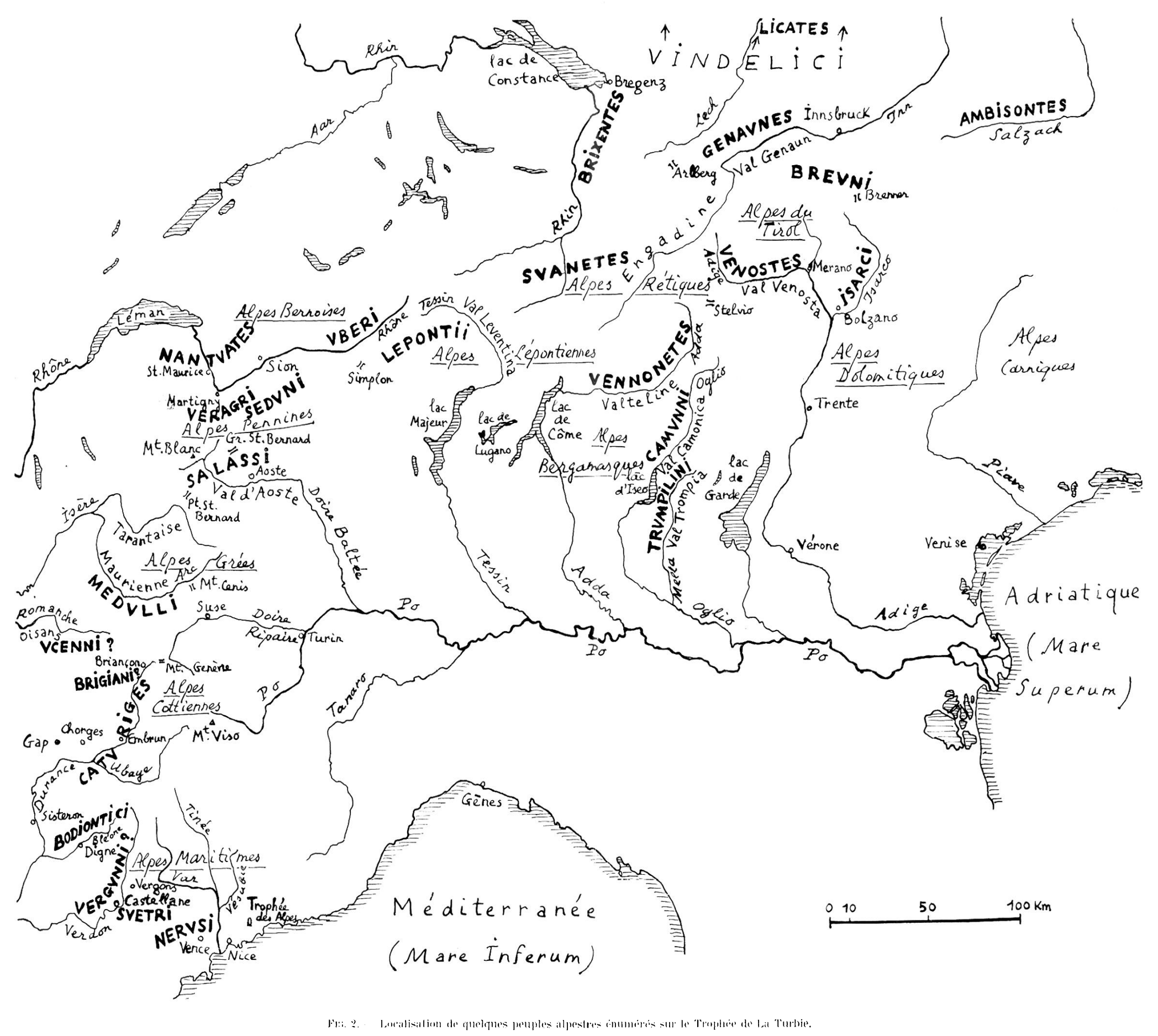

